\title{
Study on the Optimization of Multi-interval Relay Transportation Line
}

\author{
Zhongjun MA \\ Department of Transportation Service Bengbu Automobile NCO Academy Bengbu, Anhui Province, China \\ ma_vsjp@163.com
}

\begin{abstract}
The transportation route optimization problem is the important content of the military transportation guarantee decision-making, has the important influence to the transportation safeguard benefit. This paper first proposes the relay transportation problem based on the mode of multi interval transportation route optimization, and then establish the corresponding mathematical model optimization; finally, using dynamic programming along estimate algorithm is verified.
\end{abstract}

Index Terms -Multi-interval, Relay Transportation, Optimization.

\section{Introduction}

The relay transportation is one of the common methods for the military transportation. According to the different needs for goods and different characteristics of the enemy in wartime, the goods are needed to implement long-distance supplies sometimes. The whole distance from the point of departure to the end of transport line is divided into multiple sections when the relay points are determined. There is a plurality of routes available between each two adjacent relay points (i.e. interval). Because of the differences of the enemy, road conditions, traffic, the time and cost spent in different routes in each interval are not equal, which will be determined from the overall benefit of the interval of the route. The rationality of transportation route choice has a direct impact on the effectiveness of the transportation security and the economic effect.

\section{Question}

There are a number of material needs to be shipped from the rear to a line of troops. Due to the transport distance is far, road condition is complex, according to the way service support security settings and the threat of enemy, set up along the necessary transportation relay point and the relay node materials channel is divided into multiple interval, each interval have multiple feasible routes, the survey can determine the time and financial costs of each route. In the case of meeting the demand of time, choose the route of transportation routes, so that total cost is the least. How to choose the route available of each interval belongs to the problem of multi - stage decision.

\section{Model Establishment and Algorithm Analysis}

\section{A. Conditions Supposed}

1) Relay transport is needed between the start and the final, and the relay point is determined by the higher headquarters and must be passed through.
2) On the basis of the result not to be affected, the assumption of the required cost of each relay point is 0 , and the required time is 0 .

3 ) The assumption of a total of $N-1$ transport relay points is set; the entire transport line is divided into $N$ intervals.

4) There is more than one route available for each of the intervals.

\section{B. Establish the Model}

According to the problem description and conditional hypothesis, the following model can be built:

$$
\min F=\sum_{i=1}^{N} F\left(x_{i}\right)
$$

The constraint of the model is:

$$
\text { S.t. } \quad\left\{\begin{array}{l}
\sum_{i=1}^{N} Z\left(i, x_{i}\right) \leq Z_{f} \\
i=1,2, \cdots, N \\
x_{i}=1,2, \cdots, U(i)
\end{array}\right.
$$

The meaning of each parameter in the formula:

$F$ : the total cost of the entire route from the point of departure to the end;

$N$ :the total number of intervals, $N \geqslant 1$;

$i$ : Indicates the NO. $i$ interval;

$U(i)$ : Represents the total number of routes available in the NO. $i$ interval ; interval;

$x_{i}$ : Represents the NO. ${ }^{x_{i}}$ optional route in the NO. $i$

$Z\left(i, x_{i}\right)$ : Indicates the time required for selecting the NO. ${ }^{x_{i}}$ optional route in the NO. $i$ interval;

$F\left(x_{i}\right)$ : Indicates the cost required for selecting the NO. ${ }^{x_{i}}$ optional route in the interval;

$Z_{f}$ : Represents the completion time of a task. 


\section{Algorithm Analysis}

From the analysis of model, $F\left(x_{i}\right)$ and $Z\left(i, x_{i}\right)$ are a non-linear relationship, belonging to nonlinear programming problem. The essence of the problem is how to scientifically determine the transport routes of each interval, distributing reasonably the completion time available, and finding out the least of total transportation costs. This is a typical problem of resource allocation, which can be simplified as $\mathrm{N}$ stages of the multi-stage decision-making problem and can be solved by using the dynamic programming ${ }^{[1]}$ principle and method.

Suppose: $S_{i}$ is the sum of the time of route from the zeroth to the NO. $i$ relay point, then $S_{i}$ is the state variable of the NO. $i$ stage.

Termination state $S_{N+1}=Z_{f}$

Because the termination status can be determined, it is convenient to adopt the push method ${ }^{[2]}$.

The equation of state transfer is:

$S_{i}=S_{i-1}-Z\left(i, x_{i}\right), S_{0}=0$

\section{Case Analysis}

\section{A. Data of Instance}

There are 3 relay points needed (4 intervals), when a batch of goods are shipped from the point of departure to the end of transport routes. 27.5 hours is the complete time, so the minimum cost is needed to find out within the prescribed time limit.

The time and expense of the alternate routes between the every interval are shown in the following tables.

TABLE I Optional Routes for $1^{\text {st }}$ interval

\begin{tabular}{|c|c|c|c|c|}
\hline Optional route $x_{1}$ & NO.1 & NO.2 & NO.3 & NO.4 \\
\hline Time (hour) & 10 & 8 & 9 & 11 \\
\hline Cost (thousand) & 10 & 15 & 12 & 7 \\
\hline
\end{tabular}

TABLE II Optional Routes for $2^{\text {nd }}$ interval

\begin{tabular}{|c|c|c|c|c|c|}
\hline Optional route $x_{2}$ & NO.1 & NO.2 & NO.3 & NO.4 & NO.5 \\
\hline Time (hour) & 8 & 7 & 6 & 9 & 11 \\
\hline Cost (thousand) & 17 & 20 & 21 & 16 & 9 \\
\hline
\end{tabular}

TABLE III Optional Routes for $3^{\text {rd }}$ interval

\begin{tabular}{|c|c|c|c|}
\hline Optional route $x_{3}$ & NO.1 & NO.2 & NO.3 \\
\hline Time (hour) & 5 & 7 & 6 \\
\hline Cost (thousand) & 9 & 6 & 8 \\
\hline
\end{tabular}

TABLE IV Optional Routes for $4^{\text {th }}$ interval

\begin{tabular}{|c|c|c|c|}
\hline Optional route $x_{4}$ & NO.1 & NO.2 & NO.3 \\
\hline Time (hour) & 8 & 6 & 7 \\
\hline Cost (thousand) & 5 & 8 & 6 \\
\hline
\end{tabular}

\section{B. Model Solution}

According to the previous analysis, ${ }^{Z_{f}}=27.5, N=4$

$$
\min F=\sum_{i=1}^{4} F\left(x_{i}\right)
$$

$$
\text { S.t. } \quad\left\{\begin{array}{l}
\sum_{i=1}^{4} Z\left(i, x_{i}\right) \leq 27.5 \\
i=1,2,3,4 \\
x_{i}=1,2, \cdots, U(i) \\
U(1)=4, \quad U(2)=5, \quad U(3)=3, \quad U(4)=3
\end{array}\right.
$$

According to the $\mathrm{N}$-stage dynamic programming principle of the push solution, then

$$
\min F=48
$$

$$
\left\{\begin{array}{l}
x_{1}=3 \\
x_{2}=3 \\
x_{3}=1 \\
x_{4}=3
\end{array}\right.
$$

$$
\text { And } \quad \sum_{i=1}^{4} Z\left(i, x_{i}\right)=27
$$

So the result of optimization (i.e. the minimum running cost of the general line) is 48 thousands Yuan.

The optimal transport routes are: the first interval, the route No. 3 is chosen; the second interval, the route No. 3 is chosen; the third interval, the route of. No. 1 is chosen; the forth interval, the route of. No. 3 is chosen. The operating time of the total line is 27 hours. Compared with the 27.5 hours of time limit, there is 0.5 hour for free.

\section{Concluding Remarks}

The relay routing optimization problem has different models and algorithms under different conditions. The model of this paper mainly studies resources allocation based on the limit of time, which makes the balance between the time and the cost, which is important for solving the long-distance relay transportation problem with realistic significance.

\section{References}

[1] Wang Xiaohua, "Application of dynamic programming in resource allocation," Journal of Jingmen Vocational College, vol. 22, no.6, pp. 84-86, July 2007.

[2] "operations research" compiling team, Operations research: Tsinghua University press, 1997, pp.194-210. 